\title{
VIRTUAL BRONCHOSCOPY FOR DETERMINING THE SIZE OF THE CHANGES IN CENTRAL LUNG CARCINOMA
}

\author{
M. Mitev* \\ Department of Medical Physics, Biophysics, Roentgenology and Radiology, Faculty of Medicine, \\ Department of Diagnostic Imaging, University Hospital 'Prof. St. Kirkovich', \\ Trakia University, Stara Zagora, Bulgaria
}

\begin{abstract}
Introduction: The size of the changes is important for determining the invasiveness of the process and the stage of development of the neoplastic processes of the lung. The use of the method of virtual bronchoscopy as a diagnostic method in central lung carcinoma provides non-invasive visualization, but also abilities to measure changes. Purpose: The study aims to present the results of the application of the VB method in determining the size of the changes in patients with central lung carcinoma.

Methods: 130 patients, diagnosed with central carcinoma, went VB method, using 64 MDCT Siemens Definition AS. The criteria size of the changes in different localization (right or left) and sex (women, men) was considered in the work.

Results: 98 men and 32 women, aged 26-83 years, diagnosed with central lung cancer were examined using the method of virtual bronchoscopy, in which the size of the changes was monitored. In central right carcinoma of men, the formations in CT ranged in average from 5 to $68 \mathrm{~mm}(36.5 \pm 18.62)$ and the largest number of findings were of $21-43 \mathrm{~mm}(44.5 \pm 14)$ in length. The length of the changes in men with central left carcinoma ranged from $0.45 \mathrm{~mm}$ to $31 \mathrm{~mm}(12.7 \pm 10.59)$. The length of the changes in women with central right carcinoma was from 0.44 to $1.86 \mathrm{~mm}(6.45 \pm 6.83)$, and in those with left localization - from 0.6 to $13.1 \mathrm{~mm}(10.35 \pm 6.74)$. In men, all six groups of stenoses were presented, most of them with sizes over $20 \mathrm{~mm}$, followed by those with sizes below $3 \mathrm{~mm}$. In women, stenoses were presented in only 3 groups, most of which were less than $3 \mathrm{~mm}$ in size. A significant relation was found between the sex of the studied patients with central carcinoma and the length of the stenoses. Sex was a determining factor for the length of the stenoses $\left(\chi^{2}=212.77, \mathrm{p}=0.0000\right)$.

Conclusions: Virtual bronchoscopy allows accurate metry in all planes. The magnitude of the measured changes is important for determining the invasiveness of the process. The results obtained in VB should always be interpreted with axial and multiplanar images.
\end{abstract}

Key words: central carcinoma, lung, size of the changes, virtual bronchoscopy

\section{INTRODUCTION}

Lung cancer is one of the most common causes of death in the world $(1,2)$. Virtual bronchoscopy (VB) is a modern non-invasive method for diagnosing and evaluating the tracheobronchial tree, which achieves higher detection of high-grade stenoses and visualization of poststenotic areas compared to

*Correspondence to: D-r Mitko Atanasov Mitev, D.M., Department of Medical Physics, Biophysics, Roentgenology and Radiology, Faculty of Medicine, Department of Diagnostic Imaging, University Hospital "St. Kirkovich”, Trakia University - Stara Zagora, 6000 Stara Zagora, Bulgaria, Phone number: +359887 706 079, Email: mitev.mitko69@gmail.com conventional methods of examination (3-5). VB is a promising method because it makes it possible to visualize changes in early-stage lung cancer (6). VB is a very sensitive and specific diagnostic method of high clinical significance in the assessment of centrally located lung tumours $(7,8,1,2)$. VB is able to assess bronchial stenoses and obturations caused by both endoluminal pathology (tumour, mucus, foreign bodies) and external compression (anatomical structures, tumour, lymph nodes) etc., leading to accurate diagnosis and treatment $(9,10,4,5)$. The aim of the present study is to present the results of the application of the VB method in determining the size of the changes in patients with central lung carcinoma. 


\section{MATERIAL AND METHODS}

This study included 152 patients, aged 26 to 83 years that went VB method with MDCT. Patients had been previously diagnosed with the FB method. Patients were divided into four age groups (Table 1). 130 patients were diagnosed with central carcinoma. Twenty-two patients had negative results and were diagnosed with other diseases. The VB study was conducted using 64 MDCT Siemens Definition AS.
MITEV M.

Studies were performed with optimized standard protocols by using different slice thickness, current strength, voltage, etc. Scanning was done with breath-holding for about 10 seconds, automatically adjusted, with contrast enhancement or natively. The criteria size of the changes in different localization (right or left) and sex (women, men) was considered in the work.

Table 1. Patient distribution according to diagnosis, age and sex

\begin{tabular}{|l|l|l|}
\hline \multicolumn{1}{|c|}{$\begin{array}{c}\text { Diagnosis } \\
\text { Age group (yrs) }\end{array}$} & $\begin{array}{l}\text { Male, } \\
\text { number }\end{array}$ & $\begin{array}{l}\text { Female, } \\
\text { number }\end{array}$ \\
\hline $26-35$ & 1 & 1 \\
\hline $36-55$ & 4 & 4 \\
\hline $56-75$ & 69 & 20 \\
\hline$>75$ & 4 & 7 \\
\hline Total: 130 & 98 & 32 \\
\hline
\end{tabular}

\section{Statistical analysis}

The significance of changes was assessed by the $\chi^{2}$ test. In all cases of variation in the values of the compared criteria, rank and mean \pm SD were defined. The software products MS Excel (Microsoft 2010), Statistics 10 were used on statistical data processing (11).

\section{RESULTS}

The size of the VB findings along the bronchi was directly dependent on the CT characteristics of the tumour formations. The size, shape, outline and position of the findings were different, which directly affected the changes that were found in VB. In central right carcinoma of men, the formations in CT varied on average from 5 to $68 \mathrm{~mm}(36.5 \pm 18.62)$ and the largest number of findings were $21-43 \mathrm{~mm}$ $(44.5 \pm 14)$ in length. The shapes were different - oval, irregular and lobular, causing stenoses different in shape and size.

In men with central right carcinoma of the second age group, the oval shape, the irregular polygonal shape with or without spicules predominated. The dimensions of the findings in CT examination were from 14 to $68 \mathrm{~mm}$ (41 \pm 16.02 ) and with the induction of different degrees of stenoses and different types of stenoses - concentric, eccentric or mixed. The dimensions with a length of 17-29 mm $(23 \pm$ 3.89) had the largest number of changes in CT finding in axial projection. In patients of the third age group, the size of the findings in the CT examination was of 23-62 mm (42.5 \pm 11.69). By VB, the length of the changes measured in the bronchi ranged from 0.14 to a $50 \mathrm{~mm}$ long lesion $(17.54 \pm 13.57)$ with tracheal involvement.

The findings relating to the changes in the bronchi gave different degrees of stenoses and length of the changes up to $0.7-17 \mathrm{~mm}(8.85 \pm$ 11.53 ) in the area of the main bronchi and larger - up to $4.0 \mathrm{~mm}$ in the area of the trachea.

In FB, the size of the formations could not be accurately determined by indicating varying degrees of stenosis or obliteration of the bronchial lumen.

The length of the formations established by VB in men with central left carcinoma of the third age group of the axillary $\mathrm{CT}$ images ranged from 12.2 to $83 \mathrm{~mm}(47.6 \pm 35.4)$. The shape of the formations was different - from regular oval to irregular, multi-arc or polycyclic; the outlines were sharp and unsharp, with the presence of hyperdense spicules. The registered larger findings in CT showed the presence, in most of them, of spiculoid hyperdense strands or hypodense changes centrally. Bronchial obturations were registered in 5 of 34 patients $(14.71 \%)$, and in one of them the changes were bilateral.

The following were the changes of the men of the second age group, characterized by irregularly shaped stenoses with formations at CT of 13-32 $\mathrm{mm}(22.5 \pm 9.5)$. In one of the patients complete obturation was found, and in all the others stenoses of I-III degree prevailed. 
MITEV $M$.

The changes in patients of the fourth age group, registered with central left carcinoma, had the size of the formations in the CT examination of 14.7-83 $\mathrm{mm}(48.85 \pm 34.15)$. Stenoses varied from I to III degree, with the same number of those from III and those from I-II degree.

In summary, the length of reported changes in men with central left carcinoma ranged from 13 to $83 \mathrm{~mm}(48 \pm 35)$. The length of changes in virtual bronchoscopy ranged from $0.45 \mathrm{~mm}$ to $31 \mathrm{~mm}(12.7 \pm 10.59)$.
No measurement was performed on FB in most patients. Single changes from stenoses and obturations without specific dimensions, but definable according to the width of the bronchoscope and the residual length of the bronchoscope, were reported.

The ability to perform measurement in VB is one of its biggest advantages, but it should be borne in mind that in VB accurate assessment should always be performed by comparing the dimensions with axial images and multiplanar reconstructions (Figures 1, 2) .

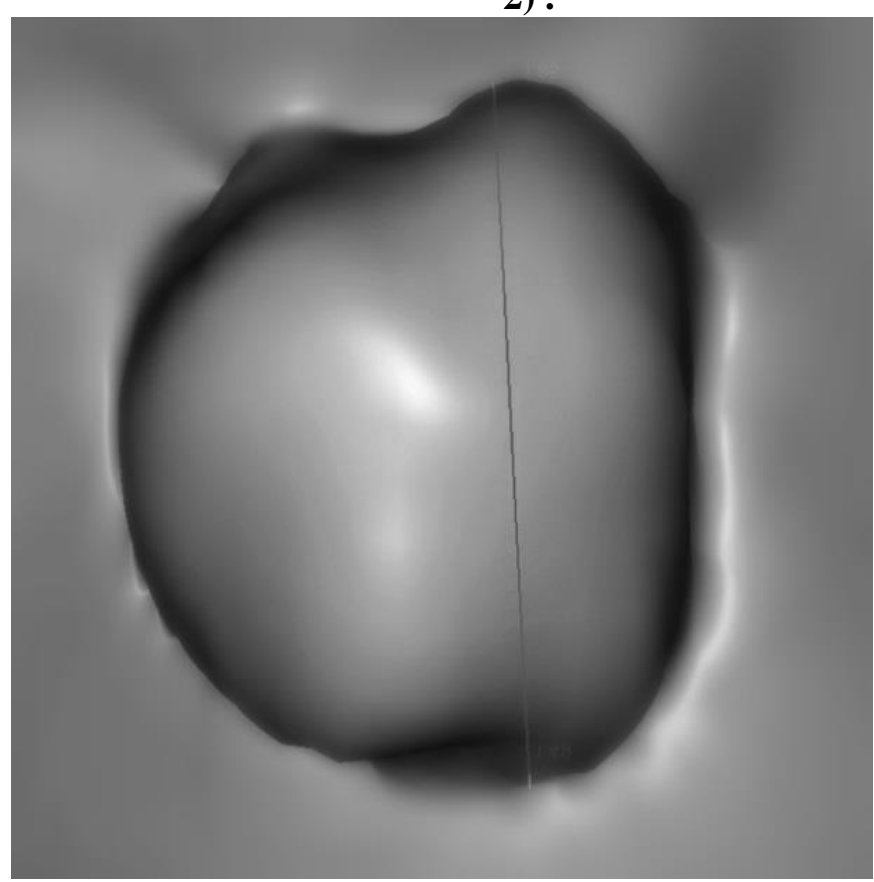

Figure 1. Measurement of lesions in VB, length (mm)

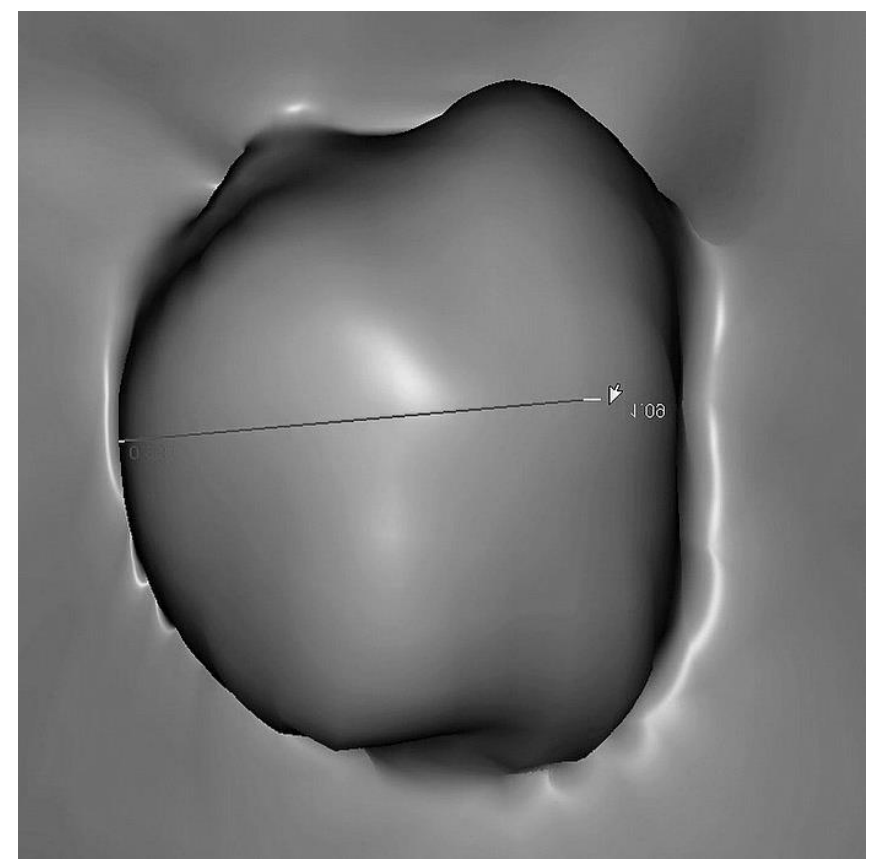

Figure 2. Measurement of lesions in VB, width (mm) 
In $\mathrm{VB}$ examined women with central right localized carcinoma, the CT size of the finding in the axial plane, for patients in the third age group, was $0.44-45 \mathrm{~mm}(22.72 \pm 22.28)$. The length of the changes in VB was from 0.44 to $1.86 \mathrm{~mm}(6.45 \pm 6.83)$. Eccentric and concentric stenoses were found in $90 \%$ of patients and grade I-III stenoses, and in one patient complete bronchial obturation. The results of the second and fourth age groups showed stenoses of I-II degree with CT sizes of the processes up to $26 \mathrm{~mm}$. In the first age group, one patient with bronchial obliteration was identified.

In summary, CT axial dimensions were 26-44 $\mathrm{mm}(35 \pm 5.63)$. The length of the changes in VB was expressed by stenotic areas to varying degrees and areas of obturation. In all women, changes associated with stenoses were observed $(100 \%)$, with a predominance of grade I-II. In $10 \%$ the stenoses were of the third degree with complete obturation of the bronchi. The most severe changes were in the third age group of women over the age of 60 . In FB, changes from stenoses without specific measurements were reported.
MITEV M.

The changes detected by VB in women with central left carcinoma were mainly in patients of the third age group. The length of the changes in VB was of $0.6-13.1 \mathrm{~mm}(10.35 \pm 6.74)$. No measurements were found from FB for women with central left carcinoma. The findings referred to the changes in the bronchi in patients with central carcinomas diagnosed with VB, gave different degrees and shape of stenoses and length of changes up to 0.3-0.7-17 mm (6 9.53 ) in the area of the main bronchi and larger - up to $43 \mathrm{~mm}$ in the area of the trachea. In FB, the type and grade of bronchial stenoses or bronchial obturations were described. As with men the indicators were smaller and very few in number.

The number of stenoses in size in men and women with central carcinoma in total could be assigned to 6 groups (according to $\mathrm{Li}$ (12), as amended): less than $3 \mathrm{~mm}$ in size; from 3.1 to 5 $\mathrm{mm}$; from 5.1 to $10 \mathrm{~mm}$; from 10.1 to $15 \mathrm{~mm}$; from 15.1 to $20 \mathrm{~mm}$; over $20 \mathrm{~mm}$. In men, six groups of stenoses were presented, most of them with sizes over $20 \mathrm{~mm}$, followed by those with sizes less than $3 \mathrm{~mm}$. In women, stenoses were presented in only 3 groups, most of which were less than $3 \mathrm{~mm}$ in size (Figure 3).

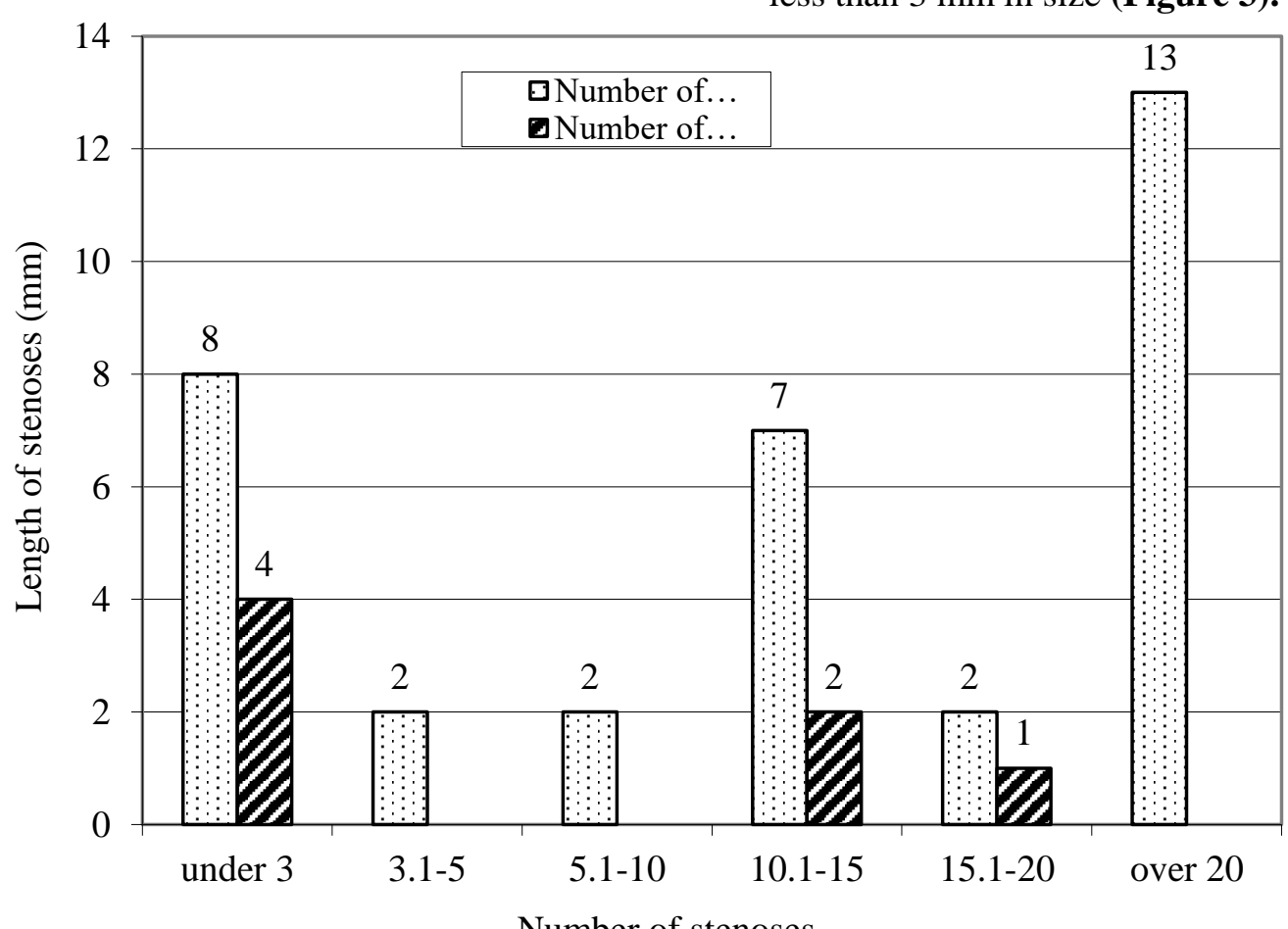

Figure 3. Groups according to the length of the stenoses (average for men - 0.14-50 mm (15.66 \pm 12.51$)$; average for women $-0.44-16 \mathrm{~mm}(6.45 \pm 6.83)$

A significant relation was found between the sex of the studied patients with central carcinoma and the length of the stenoses. Sex is a determining factor for the length of the stenoses $\left(\chi^{2}=212.77, \mathrm{p}=0.0000\right)$. 


\section{DISCUSSION}

A great advantage of the $\mathrm{VB}$ method is the ability to make measurements of both the length and width of the lumen, the size of the changes of noticeably enlarged lymph nodes or neoplastic formations. Determining the width of the lumen makes it possible to grade the stenoses (I, II, III degree), thus measuring the severity of the neoplastic infiltrations. The advantage of VB is that prestenotic changes, stenosis, and post-stenotic changes can be measured, moreover, that the measurements made in the changed areas are transformed by software in the made multiplanar reconstructions. This greatly supports the clinical practice for the direction of treatment and for possible interventions in the area of change. The altered and stenosed areas in the FB are determined accurately and correctly on the bronchoscope with the metry and the presumed result of the length and width of the bronchoscope used.

Notwithstanding the advantage of VB for the ability to determine the magnitude of the changes, it should be borne in mind that the method is virtual and the results should always be interpreted with axial and multiplanar images. In all studied patients with central carcinoma, both men and women, a measurement of the size of the lesion was made and the changes were compared with the changes and measurements in the performed VB. For example, large exobronchial neoplasms with infiltration give eccentric or concentric stenoses to several branches with changes in the length and extent of the stenoses. In $\mathrm{FB}$, its redness is pointed out as an advantage, and as a disadvantage - the determination of the size of the stenosis according to the size of the bronchoscope. FB does not allow accurate metrics of changes.

The size of the changes in central carcinomas is of 6-44 $\mathrm{mm}$. According to the results obtained for central carcinoma, the localization (left, right) is a significant factor for the length of stenoses in men $\left(\chi^{2}=268.05, \mathrm{p}=0.0000\right)$ and in women $\left(\chi^{2}=14.37, \mathrm{p}=0.0024\right)$.

Summers et al (13) state that $90 \%$ of bronchi (up to the third generation) measurable by multiplanar CT transformations are also measurable by VB. Bronchial diameters measured by VB are up to one $\mathrm{mm}$ from those measured by the multiplanar reconstructions of CT data. A number of authors (14-15 and others) point out that VB images are visually accurate and analytically correct, i.e. allow accurate measurements, diagnosis and treatment. Çeşme (2) studied the efficacy of VB in patients with central lung carcinoma. The author points out that VB should be used only in certain patients due to the longer duration of diagnosis, compared to multislice $\mathrm{CT}$, because of the fact that post-processing is performed and is not related to the patient's radiation exposure. Clinicians should select cases by assessing tumour characteristics (size, location) and patient characteristics (age, impairment of lung function) to select the most appropriate endoscopic technique (16). According to the results discussed in this study, the VB method is particularly suitable for determining the location and size of tumours. The magnitude of the measured changes is important for determining the invasiveness of the process. Depending on the size (length) of the altered areas in the trachea and main bronchi, VB may indirectly indicate the stage of carcinoma. Thus, according to Asano et al (17), $20 \mathrm{~mm}$ lesions in the area of the trachea and main bronchi point directly to the T3 stage of the neoplasms. This is supported by the ability to determine changes of lymph nodes and this should always be combined with axial images and multiplanar reconstructions. According to Ferretti et al. (18), the assessment of the length of stenoses and the adjacent tissues requires simultaneous display of the multiplanar transformations.

\section{CONCLUSION}

VB makes it possible to measure both the length and width of the lumen, the size of the changes from noticeably enlarged lymph nodes or neoplastic formations. The localization of the changes (left, right) is significant for the length of the stenoses in both sexes. Virtual bronchoscopy allows accurate metry in all planes. The magnitude of the measured changes is important for determining the invasiveness of the process. The results obtained in VB should always be interpreted with axial and multiplanar images.

\section{REFERENCES}

1. Leong, St., Shaipanich, T., Lam St. and Yasufuku K., Diagnostic bronchoscopy current and future perspectives. J Thorac Dis, 5(S5):S498-S510, 2013.

2. Çeşme, D. H., Effectiveness of Virtual Bronchoscopy in Evaluation of Centrally Located Lung Cancer. Bezmialem Science, 9(2):226-30, 2021. 
3. Röttgen, R., Schürmann, D., Pinkernelle, J., Herzog, H., Lopez-Häninnen, E., Lehmkuhl, L., Lorenz, M., Hothan, T., Felix, R. and Schröder, R. J., Detektion von Atemwegsstenosen: Vergleich der virtuellen mit der flexiblen Bronchoskopie. Fortschr Röntgenstr, 177(3):338-343, 2005.

4. Mitev, M,A., Virtual bronchoscopy with Multidetector Computer Tomography. PhD Thesis. Stara Zagora: Trakia University, 2017.

5. Mitev, M. A., Obretenov, E. D. and Valchev, D. G., Localization and shape of stenoses in central lung carcinoma - sensitivity and precision of MDCT VB and FB. Acta Clinica Croatica, 59(2): 252-259, 2020.

6. Kamath, A. V. and Chhajed P. N., Role of Bronchoscopy in Early diagnosis of Lung cancer. Ind $J$ of Chest Dis Allied Sci, 48:265269, 2006.

7. Radwan-Rhrenschef, P. and Burakowska, B., Virtual bronchoscopy versus bronchofiberoscopy - a comparison of diagnostic value in assessment of central lung tumours. Pneumonologia i Alergologia Polska, 80(2):127-139, 2012.

8. Kotlayrov, P.M., Temirhanov, S.Z., Flerov, K.E., Gombolevskii, V.A., Chernychenko, N.V., Nudnov, N.V. and Solodkiy, V.A., Virtual bronchoscopy in the diagnosis of lung cancer, in the assessment of its spread and in monitoring of post-operative changes. Newspaper RSCX-RR MH RF N13, 2013.

9. De Wever, W., Bogaert, J., Verschakelen, J. A., Virtual Bronchoscopy: Accuracy and Usefulness - An Overview. Semin Ultrasound CT MRI, 26:364-373, 2005.

10.Bairac, V., Sofroni, M., The first experience of application spiral computer tomography (SCT) in oncology. Book of Extended
MITEV M.

Synopses, 42-43, IAEA-CN-146/133P, QANTRM, Vienna, 2006.

11.StatSoft Inc., 2011. STATISTICA (data analysis software system), version 10 . www.statsoft.com

12.Li, F., Sone, Sh., Abe, H., MacMahon, H. and Doi, K., Malignant versus Benign Nodules at CT Screening for Lung Cancer: Comparison of Thun-Section CT Findings. Radiology, 233:793-798, 2004.

13. Summers, R. M., Feng, D. H., Holland, S. M. and Shelhamer, J. H., Virtual bronchoscopy: segmentation metod for real-time display. Radiology, 200(3): 857-862, 1996.

14.Summers, R. M., Selbie, W. S., Malley, J. D., Pusanik, L. M., Dwyer, A. J., Courcoutsakis, N. A., Shaw, D. J., Kleiner, D. E., Sneller, M. C., Langford, C. A., Holland, S. M. and Shelhamer, J. H., Polypoid lesions of airways: early experience with computer-assisted detection by using virtual bronchoscopy and surface curvature. Radiology, 208(2), 1998.

15.Ferguson, J. S. and McLennan G., Virtual Bronchoscopy. Proc Am Thorac Soc, 2:488491, 2005.

16.Scarlata, S., Fuso, L., Lucantoni, G., Varone, F., Magnini, D., Incalzi, R. A. and Galluccio, G. The technique of endoscopic airway tumor treatment. J Thrac Dis, 9(8):2619-2639, 2017.

17.Asano, F., Eberhardt, R. and Herth, F., Virtual Bronchoscopic Navigation for peripheral Pulmonary Lesions. Respiration, 88:430-440, 2014.

18.Ferretti, G. R., Knoplioch, J., Bricault, I., Brambilla, Ch. and Coulomb, M., Central airway stenoses: preliminary results of spiral-CT-generated virtual bronchoscopy simulations in 29 patients. Eur Radiol, 7:854-859, 1997. 